\title{
Olfactory ensheathing cell tumor: A case report and review of the literature
}

\author{
QINGCHUN MU ${ }^{1,2}$, HAIJUN GAO $^{1}$, PENGFEI LIU $^{1}$, XITONG HU $^{1}, \mathrm{XU}_{\text {ZHENG }}{ }^{1}$, PEIWEN LI $^{1}$, \\ TING LEI ${ }^{1}$, YUXUE SUN ${ }^{1}$, GANG ZHAO ${ }^{1}$, HAIYAN HUANG ${ }^{1}$ and XINYU HONG ${ }^{1}$ \\ ${ }^{1}$ Department of Neurosurgery, The First Hospital of Jilin University, Changchun, Jilin 130000; ${ }^{2}$ Department of Neurosurgery, \\ Hongqi Hospital of Mudanjiang Medical University, Mudanjiang, Heilongjiang 157011, P.R. China
}

Received June 13, 2014; Accepted February 13, 2015

DOI: $10.3892 / 01.2015 .3042$

\begin{abstract}
Olfactory ensheathing cell tumors (OECTs) are rare malignancies that occur in the anterior fossa. Little is known with regard to their clinical, radiological and immunohistochemical characteristics. The current study reports the case of a 20-year-old male with an OECT, who presented with a severe headache and a generalized convulsion. Cranial magnetic resonance imaging revealed heterogeneous enhancement of the tumor in the left anterior fossa. Complete removal of the tumor via a left frontal craniotomy was conducted, which relieved the patient's headache; however, this resulted in left-sided anosmia that remained during the two-month follow-up. The tumor was immunopositive for vimentin and S-100, and immunonegative for epithelial membrane antigen and Leu-7. The clinical data, imaging features, intraoperative findings and immunohistochemical characteristics of an additional seven cases reported in the literature are also reviewed and analyzed, providing important clinical information for the pre-operative diagnosis and intraoperative removal of OECTs.
\end{abstract}

\section{Introduction}

Olfactory ensheathing cell tumors (OECTs) are rare malignancies of the anterior fossa that share similar clinical and radiological features with olfactory groove schwannomas (OGS) (1). Forming a pre-operative diagnosis is challenging due to these similarities, which include anosmia. It is also necessary to differentiate these malignancies from common meningiomas in the anterior fossa (2). OECT was first identified as a distinct entity by Yasuda et al in 2006 (1), and only

Correspondence to: Dr Haiyan Huang or Dr Xinyu Hong, Department of Neurosurgery, The First Hospital of Jilin University, 71 Xinmin Avenue, Changchun, Jilin 130000, P.R. China

E-mail: huanghy@jlu.edu.cn

E-mail: 3294051@qq.com

Key words: olfactory groove schwannoma, immunohistochemistry, diagnosis, olfactory ensheathing cell tumor seven cases have been reported to date (1,3-8). Therefore, little information is available with regard to the clinical, radiological and immunohistochemical characteristics of OECTs (5). The current study presents the first reported case of OECT in China, which occurred in a 20 -year-old male. In addition, the seven known cases are reviewed, with a summary of the clinical manifestations, imaging characteristics, intraoperative findings and immunohistochemical features of OECTs. This provides important clinical information for the pre-operative diagnosis and intraoperative removal of OECTs.

\section{Case report}

A 20-year-old male was admitted to The First Hospital of Jilin University (Changchun, Jilin, China), after presenting with a severe headache lasting for one day and a generalized convulsion, during which the patient lost consciousness for $30 \mathrm{~min}$. The medical history included intermittent headaches for four years, which had been treated with painkillers as required. Upon examination, no neurological deficits were identified. Olfactory function was normal and no stigmata of neurofibromatosis were observed.

Cranial magnetic resonance imaging (MRI) revealed a round, patchy mass of $3.4 \times 2.6 \times 5.0 \mathrm{~cm}$ in the left anterior fossa, with long T1 and T2 signals. The lesion was hypointense on the fluid-attenuated inversion recovery sequence. Heterogeneous enhancement and necrosis of the tumor were observed following intravenous gadolinium injection (Fig. 1). Abnormal long $\mathrm{T} 1$ and $\mathrm{T} 2$ signals were observed in the tumor-adjacent tissues without enhancement. The lateral ventricles and third ventricle were enlarged with a right and posterior shift of the midline. Abnormal long T2 signals were observed in the thickened mucosa of the bilateral ethmoidal sinuses. The diagnosis was of an occupying lesion (possible meningioma) in the left anterior fossa.

The patient was placed under general anesthesia and a left frontal craniotomy was performed via a coronal incision. The tumor was located on the base of the left anterior fossa, posteriorly extending into the anterior clinoid process. The tumor was slightly adherent to the dura mater of the anterior fossa, and was surrounded by the arachnoid membrane. The tumor had grown toward the left olfactory groove and compressed the left frontal cortex. The tumor presented with a greyish-red 


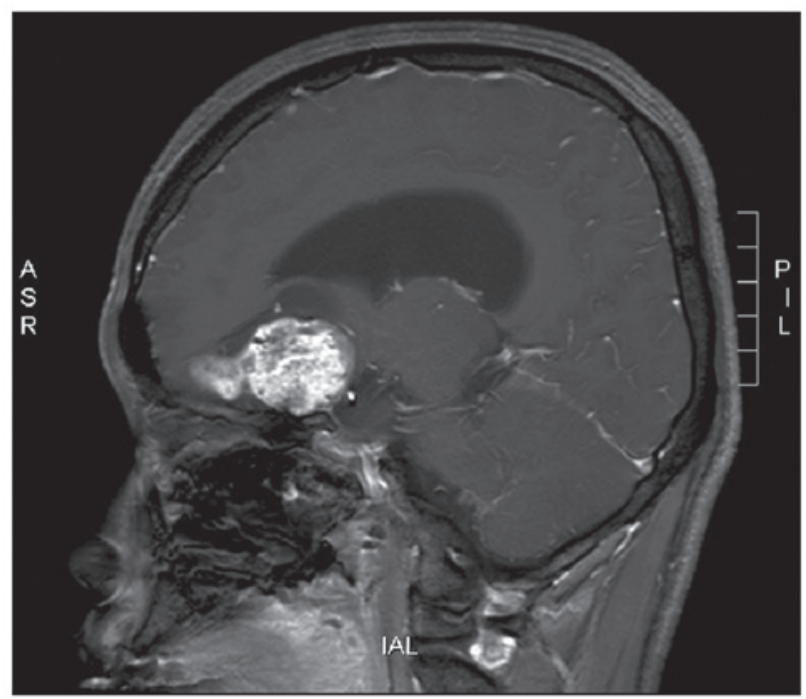

Figure 1. Cranial magnetic resonance imaging revealing a mass in the left anterior fossa, with long T1 and T2 signals. Heterogeneous enhancement of the tumor was observed following intravenous gadolinium injection. The lesion exhibited a clear boundary with the surrounding tissues.

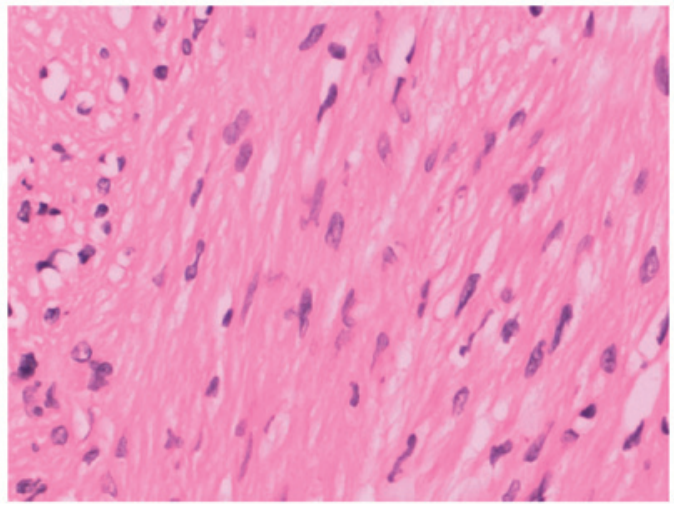

Figure 2. Hematoxylin and eosin staining revealing a tumor composed of spindle-shaped cells with elongated nuclei, arranged in compact fascicles or fibrous cords (magnification, $\mathrm{x} 400$ ).

appearance, cystic necrosis and a rich blood supply, with a rubbery encapsulation creating a distinct boundary from the surrounding brain tissue. The tumor was debulked and completely removed, as confirmed by a post-operative computed tomography (CT) scan. The left olfactory bulb and nerve tract were not observed during the surgery.

Post-operative hematoxylin and eosin (HE)-stained sections revealed that the tumor was composed of spindle-shaped cells, predominantly arranged in compact fascicles or fibrous cords, with a few cells arranged in whorls. The matrix contained bundles of mature collagen fibers parallel to the cells (Fig. 2). Immunohistochemical staining revealed that the tumor was immunopositive for vimentin and S-100, and immunonegative for epithelial membrane antigen and Leu-7 (Fig. 3), leading to a final diagnosis of OECT.

The post-operative course was uneventful, and the patient was discharged two weeks after surgery, without headache but with left-sided anosmia. The patient was in relatively good health during the two-month follow-up examination, despite
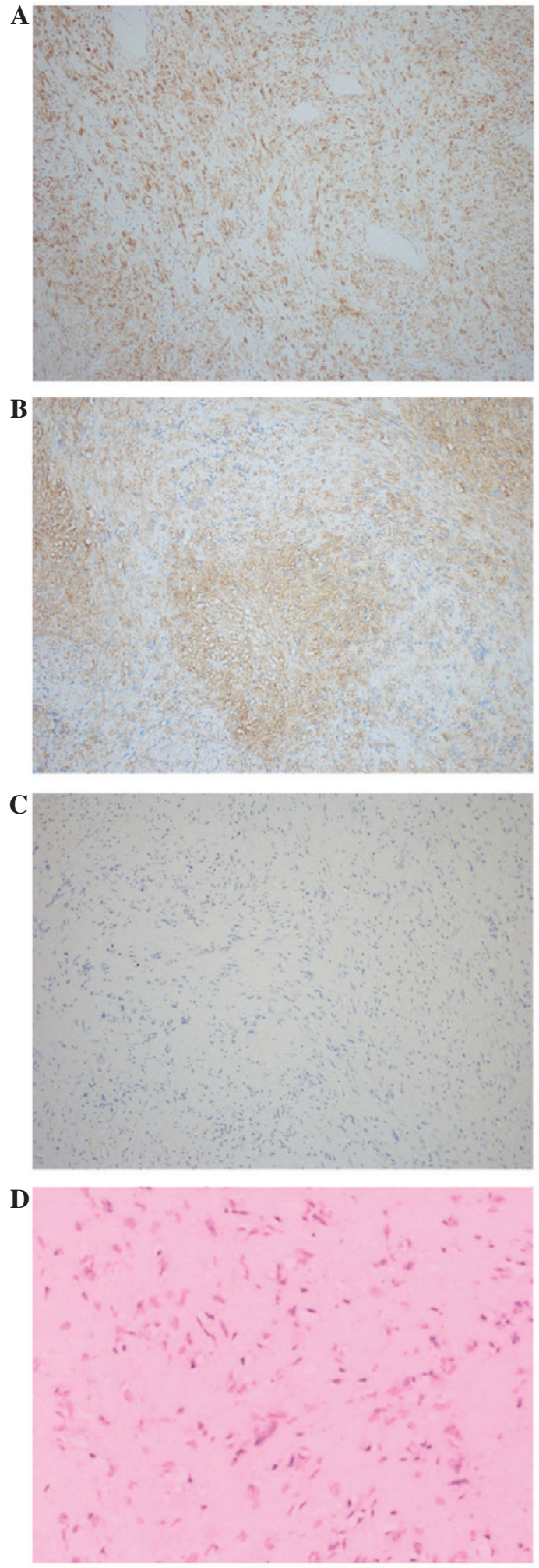

Figure 3. Immunohistochemical staining of the tumor for (A) vimentin, (B) S-100, (C) epithelial membrane antigen and (D) Leu-7 (magnification, x100).

persistent left-sided anosmia. A cranial MRI revealed no recurrence of the tumor.

\section{Discussion}

OECT was first identified as a being distinct from schwannoma based on its Leu-7 immunoreactivity (1). The current study is 
Table I. Summary of the eight published olfactory ensheathing cell tumor cases.

A, Yasuda et al, 2006 (1)

\begin{tabular}{|c|c|}
\hline Parameter & Findings \\
\hline Patient gender/age & Female/31 years \\
\hline Symptoms and signs & $\begin{array}{l}\text { Generalized convulsion; loss of olfactory sensation through the right nostril of the nose; } \\
\text { no other abnormalities }\end{array}$ \\
\hline Skin & No nevus or pigmentation \\
\hline Radiological findings & $\begin{array}{l}\text { CT: Tumor of } 6.5 \mathrm{~cm} \text { in diameter with calcified nodules located slightly right of the center } \\
\text { of the anterior skull base. MRI: Round structure exhibiting high intensity on T1WI and low } \\
\text { intensity on T2WI; finger-like extensions toward the cerebral parenchyma; heterogeneous } \\
\text { enhancement; no edema. Angiography: Avascular structure. EEG: Sporadic slow waves in } \\
\text { the right frontal area. }\end{array}$ \\
\hline Intraoperative findings & $\begin{array}{l}\text { The tumor was totally removed. The tumor was capsulated and attached strongly to the right } \\
\text { olfactory groove. The right olfactory bulb was absent, and a 5-mm bone defect was identified } \\
\text { on the right olfactory groove. The tumor extended into the sinus through the bone defect. The } \\
\text { bone defect was repaired. }\end{array}$ \\
\hline Outcome & Uneventful \\
\hline Follow-up & No recurrence during 16-month follow-up \\
\hline Electron microscopic findings & $\begin{array}{l}\text { Amoeba-like cytoplasmic process; irregular nucleus with marginally distributed chromatin; } \\
\text { abundant collagen fibers around the cells. The outer surface of the cell was covered with an } \\
\text { interrupted thin layer of basal lamina. }\end{array}$ \\
\hline Pathological findings & $\begin{array}{l}\text { Spindle-shaped cells in an interwoven pattern; distorted and twisted nuclei; wavy cellular } \\
\text { arrangement in an atypical palisading pattern. }\end{array}$ \\
\hline
\end{tabular}

B, Ippili et al, 2009 (3)

Parameter

Patient gender/age

Symptoms and signs

Skin

Radiological findings

Intraoperative findings

Outcome

Follow-up

Electron microscopic findings

Pathological findings
Findings

Male/42 years

Multiple episodes of generalized tonic clonic seizures; normal olfaction; no local neurological deficits

No neurocutaneous markers

CT: Extra-axial isodense mass in the left subfrontal region; homogenously enhanced with contrast. MRI: Isointense on T1WI and mildly hyperintense on T2WI and FLAIR.

Excision of the tumor was performed via a left frontal craniotomy. The tumor was partially removed by suction and partially fibrous, and arose from the left olfactory bulb.

Uneventful

No recurrence during the one-year follow-up

NA

Spindle-shaped cells arranged in short fascicles, palisades and whorls. The cells were spindled out with curved vesicular nuclei and ill-defined cytoplasmic margins.

C, Lin et al, 2010 (4)

Parameter

Patient gender/age

Symptoms and signs

Skin

Radiological findings

Intraoperative findings
Findings

Male/32 years

Seizure attacks for six months, with one episode resulting in a traffic accident; no neurological deficits

No abnormality or stigmata of neurofibromatosis

MRI: 3.6x3.3x3.9-cm, extra-axial well-defined mass at the left frontal base; hypointense on T1WI and isointense on T2WI; heterogeneously enhanced after intravenous gadolinium administration; intact cribriform plate; no dural tail sign; no ethmoidal sinus invasion.

The tumor was completely removed via frontal craniotomy. The tumor was greyish-white with a glistening appearance and rubbery consistency over the frontal base region. The tumor 
Table I. Continued.

Parameter

Findings
Outcome

Follow-up

Electron microscopic findings

Pathological findings was traced to the proximal part of the left olfactory tract. The olfactory bulb was not identified. Intact frontal skull base.

Uneventful

No recurrence during the six-month follow-up

Amoeba-like cytoplasmic process; irregular or indented nucleus in the center of the cell. The chromatin was evenly distributed throughout the karyoplasm, and slightly condensed below the nuclear membrane. No prominent external lamina. A layer of interrupted deposits of basal lamina at certain parts of the cellular membrane was observed. Numerous extra cellular collagen fibers were present.

Low cellular tumor with dense hyalinization in the majority of areas. Tumor cells had ovoid to elongated, normochromatic and often comma-shaped nuclei. The cells had no particular arrangement. Myxoid changes of stroma were prominent in focal areas with no necrosis, mitosis or hypercellularity. A previous hemorrhage with hemosiderin deposition was observed.

D, Darie et al, 2010 (5)

Parameter

Patient gender/age

Symptoms and signs

Skin

Radiological findings

Intraoperative findings

Outcome

Follow-up

Electron microscopic findings

Pathological findings
Findings

Female/28 years

18-month history of complex partial seizures, emotional lability, and anosmia; no increase in intracranial pressure; no visual symptoms

No cutaneous stigmata of neurofibromatosis; no family history of von Recklinghausen disease

CT: Homogenous extra-axial isodense lesion at the middle of anterior cranial fossa with a left preponderance development; bone window coronal CT scan showed skull base erosion under the tumor bulk. MRI: Mass of 4x3.5x2.5 cm; hypointense on T1WI; hyperintense on T2WI and FLAIR without surrounding edema; strong heterogeneous enhancement of the tumor after intravenous gadolinium injection.

A total excision of the tumor via a left frontal pterional craniotomy was performed. The tumor was whitish, firm and non-hemorrhagic, with erosion into the cranial base; the tumor was surrounded by arachnoiditis and connected to the anterior part of the cribriform plate and the left olfactory tract. The right olfactory tract displaced laterally and was preserved, however the left olfactory tract was removed due to close adhesion to the tumor. Bone scalloping was observed underneath the tumor; no bone defect was disclosed. The dura mater defect was repaired by a temporalis flap and fibrin glue.

Anosmia

No recurrence during the 16-month follow-up

NA

Well-circumscribed tumor respecting the arachnoid space and the cerebral tissue. The tumor showed an atypical fibrous component with a few areas of compact spindle-shaped cells and no necrosis, mitosis or hypercellularity.

E, Yamaguchi et al, 2010 (6)

Parameter

Patient gender/age

Symptoms and signs

Skin

Radiological findings

Intraoperative findings
Findings

Female/30 years

Headache for several months with right anosmia; no other neurological deficits NA

CT: Three-dimensional CT revealed erosion of the cribriform plates. MRI: Heterogeneously enhancing mass of $\sim 4 \mathrm{~cm}$ in diameter in the frontal base.

The tumor was completely removed via a bilateral frontal craniotomy. The tumor was located in the intradural, extra-axial space, attached to the right cribriform plate. 
Table I. Continued.

Parameter

Outcome

Follow-up

Electron microscopic findings

Pathological findings
Findings

Olfactory function was not restored

NA

NA

Tumor cells formed patterns of compact fascicular Antoni A areas with palisading nuclei.

F, Ogino-Nishimura et al, 2012 (7)

Parameter

Patient gender/age

Symptoms and signs

Skin

Radiological findings

Intraoperative findings

Outcome

Follow-up

Electron microscopic findings

Pathological findings
Findings

Female/41 years

Headache and loss of olfactory function

NA

CT: Lesion at the olfactory cleft that extended superiorly to the olfactory groove, with a bone defect in the skull base; the cribriform plate was elevated upward. MRI: Mass showing cystic changes, with solid portions demonstrating strong post gadolinium contrast enhancement.

Nasal endoscopy: Soft, whitish mass occupying the olfactory cleft and extending laterally to the middle meatus, with destruction of the middle turbinate in the left nostril.

Subtotal resection of the tumor via an endoscopic endonasal approach was performed. The tumor was attached, but had not invaded the internal orbital wall. The tumor was debulked with an ultrasonic surgical aspiration. The bone defect was covered with a mucoperiosteal pedicle flap.

Uneventful

No recurrence during the two-year follow-up

NA

Tumor composed of cells with eosinophilic cytoplasm and elongated or wavy nuclei with occasional symplastic changes. The mitotic index was $<1$ per 10 high-power fields. No tumor necrosis was observed, and the Ki-67 labeling index was $2 \%$.

G, Al-Ghanem et al 2013 (8)

Parameter

Patient gender/ age

Symptoms and signs

Skin

Radiological findings

Intraoperative findings

Outcome

Follow-up

Electron microscopic findings

Pathological findings
Findings

Male/49 years

Visual impairment and hyposmia

NA

CT and MRI: Subfrontal cystic extra-axial mass that eroded the right cribriform plate

A total excision of the tumor via bilateral craniotomy was performed.

NA

NA

NA

NA

$\mathrm{H}$, Present case

Parameter Findings

Patient gender/age

Symptoms and signs

Skin

Radiological findings
Male/20 years

Severe headache with a generalized convulsion; no anosmia; no neurological deficits No cutaneous stigma of neurofibromatosis

MRI: Round, patchy mass of $3.4 \times 2.6 \times 5.0 \mathrm{~cm}$ in the left anterior fossa, with long T1 and T2 signals; hypointense on FLAIR sequence; heterogeneous enhancement after intravenous gadolinium injection 
Table I. Continued.

\section{Parameter}

Intraoperative findings

Outcome

Follow-up

Electron microscopic findings

Pathological findings
Findings

The tumor was completely removed through a left frontal craniotomy via a coronal incision. The tumor extended into the anterior clinoid process, and slightly adhered to the dura mater of the anterior fossa. The tumor tended to grow toward the left olfactory groove, and had compressed the left frontal cortex. The greyish-red tumor with rich blood supply was capsulated with rubbery consistency, and clear boundaries with surrounding brain tissues. Cystic necrosis inside the tumor was observed.

Left anosmia; normal right olfactory function

No recurrence during the two-month follow-up

NA

Spindle-shaped cells were predominantly arranged in compact fascicles or fibrous cords, and a few cells were arranged in whorls. The matrix contained bundles of mature collagen fibers parallel to the cells.

CT, computed tomography; EEG, electroencephalogram; EMA, epithelial membrane antigen; FLAIR, fluid-attenuated inversion recovery; GFAP, glial fibrillary acidic protein; MRI, magnetic resonance imaging; NA, not available; SMA, smooth muscle actin; WI, weighted imaging.

the eighth reported case of OECT. Table I summarizes the clinical manifestations, imaging characteristics, intraoperative findings and immunohistochemical features of all reported cases. There appears to be no gender difference amongst the eight OECT cases, with four male and four female patients affected. The patient in the present case is the youngest from the reported cases, and the average age of the OECT patients is 34.1 years (range, 20-49 years). This is younger than that of patients with schwannomas (9).

As OECTs are located in the anterior fossa close to the olfactory nerve, olfactory dysfunction is a common clinical manifestation (1,3-8). Five of the seven cases reported in the literature presented with anosmia, including two cases with right-sided anosmia, two cases with bilateral anosmia and one case with hyposmia. However, the current patient, along with two additional cases, had normal olfaction, suggesting that olfactory dysfunction is not a necessary indicator for OECT patients. In addition, seizures occurred in four cases, including one case with a generalized convulsion (1), one case with multiple episodes of generalized tonic clonic seizures (3), one case with seizure attacks for six months (4), and one case with complex partial seizures for 18 months (5). In the present case, the patient experienced a severe headache with a generalized convulsion, and also presented with a history of intermittent headaches over four years, which may have been associated with increased intracranial pressure. Furthermore, emotional lability with complex partial seizures occurred in one patient (6), and visual impairment occurred in another case (8). No cutaneous stigmata of neurofibromatosis were observed in any of the cases, however, five cases, including the present case, reported skin conditions.

All patients in the eight reported cases underwent CT and/or MRI scans. Of the six patients for whom CT scans were conducted, a tumor with calcified nodules was observed in one patient (1), and homogenous enhancement was found in another (3). In addition, tumor invasion to the skull base and ethmoid bone was reported in four cases. In certain cases, tumor erosion of the cribriform plates or skull base was clearly visualized by three-dimensional (6) and bone window coronal (5) CT scans. Therefore, these types of CT scan are strongly recommended due to their ability to identify tumor invasion, and thus are useful for distinguishing OECT from meningioma. One study reported that the tumor appeared as an avascular structure on angiography (1). Tumors with cystic changes were described in two cases $(7,8)$, and strong post-gadolinium contrast enhancement in the solid portions of the tumor was reported in one case (7). Two cases reported that there was no edema in the tissues surrounding the tumor $(1,5)$, possibly due to the encapsulation and expansive growth of the tumors. The large diameter of the masses, ranging from 3.9 to $6.5 \mathrm{~cm}$, is likely due to the large space available in the anterior fossa, and the prolonged growth period promoted by minimal intracranial pressure symptoms. Heterogeneous enhancement of the tumor on MRI following intravenous contrast administration was reported in six cases, and no dural tail signs were observed in any of the eight cases. Tumor necrosis was visible on enhanced MRI in the present case. Enhanced MRI may therefore be useful for distinguishing OECTs from meningiomas in the anterior fossa, which commonly display homogeneous enhancement without any signs of necrosis (2). However, radiological imaging may be unable to distinguish OECT from OGS.

To date, the only method that allows the distinction of OECT from OGS is immunohistochemical staining: OECTs are immunonegative for Leu-7 and epithelial membrane antigen, and immunopositive for S-100, while OGSs are immunoreactive for Leu-7 (1). Other pathological characteristics of OECTs include the arrangement of spindle-shaped cells in fascicles, fibrous cords or whorls, with distorted nuclei arranged in a palisading pattern, as observed by HE staining. In the cases described, only one reported that the tumor had indications of a previous hemorrhage with hemosiderin deposits (4). Two cases reported electron microscopic findings, in which amoeba-like cytoplasmic processes with abundant collagen fibers around the cells were observed $(1,4)$. 
Since OECTs are located in the midline or extra-axial spaces, the majority of the tumors (7/8) were excised via unilateral or bilateral frontal craniotomies, with a subtotal incision performed via an endoscopic endonasal approach in one case (7). The tumors were described as greyish-white $(4,5)$, firm (5) or cystic (7), with less blood loss during surgery compared with OGS. However, the tumor in the present case was greyish-red with a rich blood supply and cystic necrosis. Tumor invasion into the olfactory groove, cranial base, internal orbital wall or cribriform plate was reported in four cases $(1,5-7)$, and the bone or dura mater defects were repaired using a temporalis flap and fibrin glue, or mucoperiosteal pedicle flaps $(5,6)$. Similar to two of the reported cases $(1,4)$, in the present case, the olfactory bulb and tract (left side) were not observed. In addition, the current patient exhibited no tumor recurrence at the two-month follow-up examination. Likewise, no tumor recurrences were reported in the previous cases, with a mean follow-up period of 14.8 months (range, 6-24 months).

Taken together, the results of the current case and the eight reviewed cases indicate that a diagnosis of OECT should be considered for tumors in the anterior fossa occurring in patients with a history of single or multiple seizure attacks and no cutaneous stigmata of neurofibromatosis, who present with tumor invasion into the skull base and ethmoid bone on CT scans, and heterogeneous enhancement of the tumor with no edema in surrounding tissues on MRI scans. These encapsulated tumors tend to grow toward the ethmoid bone with clear boundaries from surrounding tissue, enabling their complete removal. If the tumor invades the bone, the bone defect may be repaired by a mucoperiosteal pedicle flap and fibrin glue. Although OECTs may be diagnosed by immunostaining, further cases are required to enable further characterization of the clinical findings, imaging features and immunohistochemical properties of OECTs.

\section{References}

1. Yasuda M, Higuchi O, Takano S and Matsumura A: Olfactory ensheathing cell tumor: a case report. J Neurooncol 76: 111-113, 2006.

2. Morales-Valero SF, Van Gompel JJ, Loumiotis I and Lanzino G: Craniotomy for anterior cranial fossa meningiomas: historical overview. Neurosurg Focus 36: E14, 2014.

3. Ippili K, Ratnam BG, Gowrishankar S, Ranjan A and Lath R: Olfactory ensheathing cell tumor. Neurol India 57: 76-78, 2009.

4. Lin SC, Chen MH, Lin CF and Ho DM: Olfactory ensheathing cell tumor with neurofibroma-like features: a case report and review of the literature. J Neurooncol 97: 117-122, 2010.

5. Darie I, Riffaud L, Saïkali S, Brassier G and Hamlat A: Olfactory ensheathing cell tumour: case report and literature review. J Neurooncol 100: 285-289, 2010.

6. Yamaguchi T, Fujii H, Dziurzynski K, Delashaw JB and Watanabe E: Olfactory ensheathing cell tumor: case report. Skull Base 20: 357-361, 2010.

7. Ogino-Nishimura E, Nakagawa T, Mikami Y and Ito J: Olfactory ensheathing cell tumor arising from the olfactory mucosa. Case Rep Med 2012: 426853, 2012.

8. Al-Ghanem R, Ramos-Pleguezuelos FM, Pérez-Darosa SI, Galicia-Bulnes JM, Cabrerizo-Carvajal F and El-Rubaidi OA: Olfactory ensheathing cell tumour: case report and literature review. Neurocirugia (Astur) 24: 130-134, 2013 (In Spanish).

9. van Leeuwen BM, Borst JM, Putter H, Jansen JC, van der Mey AG and Kaptein AA: Emotional intelligence in association with quality of life in patients recently diagnosed with vestibular schwannoma. Otol Neurotol 35: 1650-1657, 2014. 